\title{
Langmuir
}

This document is confidential and is proprietary to the American Chemical Society and its authors. Do not copy or disclose without written permission. If you have received this item in error, notify the sender and delete all copies.

\section{Supramolecular organization and heterochiral recognition in Langmuir monolayers of chiral azobenzene surfactants}

\begin{tabular}{|r|l|}
\hline Journal: & Langmuir \\
\hline Manuscript ID: & la-2013-006407.R1 \\
\hline Manuscript Type: & Article \\
\hline Date Submitted by the Author: & n/a \\
\hline Complete List of Authors: & $\begin{array}{l}\text { Pulido-Companys, Alba; Universitat de Barcelona, Química Física } \\
\text { Albalat, Rosa; University of Barcelona, Physical Chemistry } \\
\text { Garcia-Amoros, Jaume; University of Barcelona, Organic Chemistry } \\
\text { Department } \\
\text { Velasco, Dolores; Universitat de Barcelona, Organic Chemistry Department } \\
\text { Ignés, Jordi; Universitat de Barcelona, Química física }\end{array}$ \\
\hline
\end{tabular}

SCHOLARONE ${ }^{m}$

Manuscripts 


\title{
Supramolecular organization and heterochiral
}

\section{recognition in Langmuir monolayers of chiral}

\section{azobenzene surfactants}

\author{
Alba Pulido-Companys ${ }^{a}$, Rosa Albalat ${ }^{a}$, Jaume Garcia-Amorós $^{b}$, Dolores Velasco $^{b}$, and \\ Jordi Ignés-Mullol ${ }^{a}$
}

${ }^{a}$ Departament de Química Física and ${ }^{b}$ Departament de Química Orgànica, Institute of Nanoscience and Nanotechnology, Universitat de Barcelona, Martí i Franquès 1, 08028 Barcelona, Spain.

\begin{abstract}
We study the self-assembly of novel azobenzene-based chiral surfactants at the air/water interface, and find that while the pure enantiomers lack the ability to organize in ordered mesophases, the racemic mixture spontaneously forms a hexatic phase at low lateral pressures, which we detect by means of Brewster angle microscopy. This work provides a so far unique example of heterochiral recognition in which the racemic monolayer is not only condensed with respect of the pure enantiomers, but causes an ordered mesophase to form. Although hexatic order vanishes at high surface pressures, long-range orientational order is regained for all compositions upon monolayer collapse, which proceeds through the formation of birefringent trilayers with a well-defined lateral microstructure, as revealed by Atomic Force Microscopy.
\end{abstract}




\section{INTRODUCTION}

The study of chiral interfaces, in particular in the context of molecular recognition, is of significant interest in material science, for instance in the development of sensors, optical filters, or catalytic surfaces ${ }^{1}$. Particular attention is deserved to insoluble surfactant monolayers self-assembled at the air-water interface that may be transferred onto solid support by means of the Langmuir-Blodgett (LB) technique ${ }^{2}$. Chiral recognition in these systems, both due to interaction within monolayer components or between monolayers and aqueous guests, has been extensively studied. ${ }^{3}$ The high specificity of the lateral interactions in such low-dimensional systems can lead to significant differences in the thermodynamics of the racemic mixture when compared to the pure enantiomers ${ }^{4}$, or may result in the spontaneous segregation of racemic mixtures into two-dimensional enantiomorphic crystals $^{5-7}$. The presence of chiral surfactants often results in the formation of submillimeter domains with chiral shape ${ }^{8}$ or molecular $\operatorname{organization}^{9,10}$, either during the isothermal condensation of ordered monolayer phases or during the collapse into stacked multi-layers. Monolayers of racemates may result in the chiral symmetry breaking of the self-assembled structures. The resulting racemic domains either feature segregated regions expressing one chirality or hinder the expression of molecular chirality altogether ${ }^{11}$. Heterochiral molecular recognition evidenced in changes in the phase behavior of racemic mixtures compared to pure enantiomers has also been reported both for low molar mass and for polymeric liquid crystals $^{12,13}$. Recently, experiments experiments with racemic mixtures of aminoacid derivatives revealed new modes of self-assembly into nanostructured previously unobserved in the pure enantiomers ${ }^{14,15}$.

Among non-biomimetic films, monolayers of surfactant molecules featuring an azobenzene moiety have been the object of significant research interest both for the 
possibility to build photosensitive films ${ }^{16}$ and for the enhanced ability of trans azobenzene molecules to statically and dynamically self-assemble at the air-water interface $^{17}$. Although azobenzene-based compounds, both surfactants and nonsurfactants, have been reported to form stable monolayers at the air/water interface, the self-assembly of ordered mesophases is restricted to very specific molecular structures ${ }^{18}$. A paradigmatic example is provided by the achiral fatty acid azobenzene surfactant $8 \mathrm{Az} 3^{19}$, which features a rich polymorphism including labile photosensitive phases ${ }^{17}$ and structures with orientational chirality that can be selected either by means of a chemical dopant of by the application of vortical stirring ${ }^{20}$. Recently, Haro et al., have reported experiments aimed at characterizing the supramolecular architechture of Langmuir monolayers and LB films of a chiral azobenzene surfactant, which we will denote $(S)-3^{\prime} \mathrm{Az} 7^{21}$. Although this compound forms stable monolayers that enable the transfer of multilayer LB films, the authors do not observe the emergence of lateral order in this system.

In this work we synthesize and study the self-assembly behavior of the two enantiomers $(R / S)$ of the chiral azobenzene fatty acid 7'Az3 (see Scheme 1). In a recent publication, we showed that these amphiphilic molecules could be employed as chiral dopants to select the otherwise random chirality of self-assembled domains of the surfactant $8 \mathrm{Az} 3$. In the current work, we wanted to explore the ability of the pure chiral analogues to self-assemble into ordered chiral structures. We find that, in contrast to the behavior of $8 \mathrm{Az} 3$, the ability of the pure enantiomers to form mesophases is hindered, presumably by the presence of the chiral center. Remarkably, a racemic mixture of the $R / S$ enantiomers of 7'Az3 does self-assemble into an ordered mesophase at low lateral pressures. Brewster angle microscopy observations reveal the presence of pinwheel 
defect configurations, which is an unambiguous evidence of the existence of hexatic order at the nanoscale ${ }^{22}$. This is a striking example of heterochiral recognition in monolayers in which a high degree of ordering is obtained through the interaction of two complementary chiral species that independently fail to organize as a mesophase. At all compositions, the chiral surfactants organize into ordered multilayers above the monolayer collapse pressure. The resulting submillimeter domains have been transferred onto engineered silica surfaces in order to analyze their configuration at the nanoscale by means of Atomic Force Microscopy, revealing a well-defined microstructure that depends on the monolayer composition.

\section{EXPERIMENTAL SECTION}

Chemicals. All reagents for surfactant synthesis were used as received without further purification. Flash chromatography was carried out over silica gel (SDS, 230-240 mesh). Anhydrous THF (Sharlau) was distilled from sodium/benzophenone. Anhydrous DMF (Fluka) was stored over activated $4 \AA$ molecular sieves under inert atmosphere. Ultrapure water from a Millipore Milli-Q system was used as the subphase for Langmuir monolayers. Spreading solutions were prepared by dissolving the surfactants in chloroform (Baker, p.a.).

Characterization of synthesized surfactants. Melting points were determined by polarized optical microscopy (POM) using a Nikon Eclipse polarizing microscope equipped with a Linkam THMS 600 hot stage and a Linkam CI 93 programmable temperature controller at a scan rate of $7{ }^{\circ} \mathrm{C} \min ^{-1}$. FT-IR spectra were registered in a Nicolet 6700 FT-IR spectrophotometer from Thermo Scientific. Electronic spectra were recorded in a Varian Cary 500E UV-Vis-NIR spectrophotometer. ${ }^{1} \mathrm{H}$ NMR (400 MHz) and ${ }^{13} \mathrm{C}$ NMR (100 MHz) spectra were collected on a Varian Mercury spectrometer. 
NMR spectra have been processed with the MestRec commercially available software. HR-MS was performed in a LC/MSD-TOF Agilent Technologies apparatus by means of the electrospray (ESI-MS) technique. Optical specific rotation was measured in a Jasco P-2000 polarimeter.

\section{Preparation and characterization of Langmuir and Langmuir-Blodgett films.}

Langmuir monolayers were spread on a thermostatic KSV-Nima Minitrough Teflon trough $\left(365 \times 75 \times 5 \mathrm{~mm}^{3}\right)$ filled with Milli-Q water. The subphase temperature was kept at $25^{\circ} \mathrm{C} \pm 0.2^{\circ} \mathrm{C}$ in the experiments reported herein by means of a water circulation bath. The system was computer-controlled by means of the provided KSV software. Monolayers were prepared by spreading a $1 \mathrm{mg} \mathrm{cm}^{-3}$ surfactant solution in chloroform. After spreading, a time interval of about 5 minutes was allowed for evaporation of the solvent. The monolayer was subsequently compressed at $6 \mathrm{~mm} \mathrm{~min}^{-1}$ (compression rate of order $10^{-2} \mathrm{~nm}^{2}$ molec ${ }^{-1} \mathrm{~min}^{-1}$ ) by means of two symmetric spring-loaded Teflon barriers which provided a leak-free enclosure. The surface pressure of the monolayers was measured using a filter paper Wilhelmy plate attached to the electrobalance included with the Minitrough system. In order to probe the mesoscale organization of the monolayers, a custom-built Brewster angle microscope (BAM) was used. This microscopy technique reveals spatial differences in the monolayer reflectivity, and spatial contrast observed in BAM micrographs depends on the local film density, thickness and, in systems featuring mesoscale ordering ${ }^{10}$, average molecular orientation. Real time microscopy images were captured by means of a Sony XC-77CE CCD camera and stored in digital format for further processing with the public domain software ImageJ. The setup was placed inside an enclosure to avoid air currents and to work in the dark. The substrates used for the Langmuir-Blodgett films, $\mathrm{SiO}_{2} \mathrm{SURF}$ Standard $\left(10 \times 10 \times 0.675 \mathrm{~mm}^{3}\right)$, were commercially obtained from Nanolane. They are 
based on a silicon wafer substrate and engineered with a sequence of inorganic layers to tune their optical reflectivity so that light with normal incidence is reflected without changing its state of polarization. The top layer is composed of $\mathrm{SiO}_{2}$, so the terminal groups are $\mathrm{SiO}_{2} / \mathrm{SiOH}$, and the rms roughness is less than $0.1 \mathrm{~nm}$. The substrates were activated with piranha solution $\left(\mathrm{H}_{2} \mathrm{SO}_{4}+\mathrm{H}_{2} \mathrm{O}_{2} 3 \div 1 \mathrm{v} / \mathrm{v}\right.$. CAUTION: Piranha solution is an extremely strong oxidant and should be handled only with the proper equipment). Langmuir films were transferred to the substrates using the KSV-Nima Minitrough dipper at a velocity of $8 \mathrm{~mm} \mathrm{~min}^{-1}$. LB films were subsequently characterized by optical microscopy using a custom-built polarizing reflection microscope, and Atomic Force Microscopy (Nanotec Electrónica SL, Cervantes AFM system), using arrow-NCR-20 cantilevers in non-contact mode, with a thickness of $4.6 \mu \mathrm{m}$, a length of $160 \mu \mathrm{m}$, and a width of $45 \mu \mathrm{m}$. Their resonance frequency is $285 \mathrm{kHz}$ approximately, and their force constant is $42 \mathrm{Nm}^{-1}$. AFM image acquisition and image processing has been performed with the aid of the software $\mathrm{WSxM}^{23}$. In the reported images, filters plane, flatten, and equalize have been applied on the raw data.

The original design of the AFM header (see Fig. S1, Supplementary Information) provides a clear view access, through beam-splitter BS2, to the cantilever on top of the sample under investigation. As is typical in this type of instruments, the original design included a polarizing beam splitter that maximizes the reflection of the polarized laser used to probe the tip deflection (red beam) but modifies the state of polarization of the light beam from the polarizing optical microscope (green beam). The ability to visualize thin aggregates on the SURF substrate relies on the use of polarized light and the observation of its reflection through a crossed analyzer. Reflection from the bare substrate is minimized this way so observed textures come from the presence of 
laterally organized thin films that modify the state of light polarization. The original polarizing beam splitter BS2 was replaced by a non-polarizing one to enhance the signal to noise ratio. With this modification we were able to obtain an accurate registry between optical observation of aggregates and the corresponding AFM scans.

Synthesis of the chiral azocompounds $(R)-7^{\prime} A z 3$ and $(S)-7^{\prime} A z 3$. The two compounds were prepared starting from 4-hydroxy-4'-methoxyazobenzene which was obtained as described previously ${ }^{24}$. The alkylation of 4-hydroxy-4'-methoxyazobenzene via the Mitsunobu's reaction with $(S)$-2-octanol and $(R)$-2-octanol in the presence of DIPAD (diisopropyl azodicarboxylate) and $\mathrm{PPh}_{3}$ in THF afforded azocompounds (S)-1 and (R)$\mathbf{1}$, respectively. The subsequent selective cleavage of the methyl ether of $(S)-1$ or $(R)-1$ with sodium ethanethiolate in DMF furnished the corresponding chiral azophenols $(\boldsymbol{S})-\mathbf{2}$ and $(\boldsymbol{R})-\mathbf{2}$ in high yield. The Williamson's alkylation of $(\boldsymbol{S})-\mathbf{2}$ and $(\boldsymbol{R})-\mathbf{2}$ with methyl-4bromobutyrate in DMF using $\mathrm{K}_{2} \mathrm{CO}_{3}$ as a base gave azo-dyes $(\boldsymbol{S})-\mathbf{3}$ and $(\boldsymbol{R})-\mathbf{3}$ which were further hydrolysed under basic conditions providing us the final chiral azoderivatives (S)-7'Az3 and (R)-7'Az3 (Scheme 1). See Supplementary Information for details of the synthesis of the different compounds present at each stage.

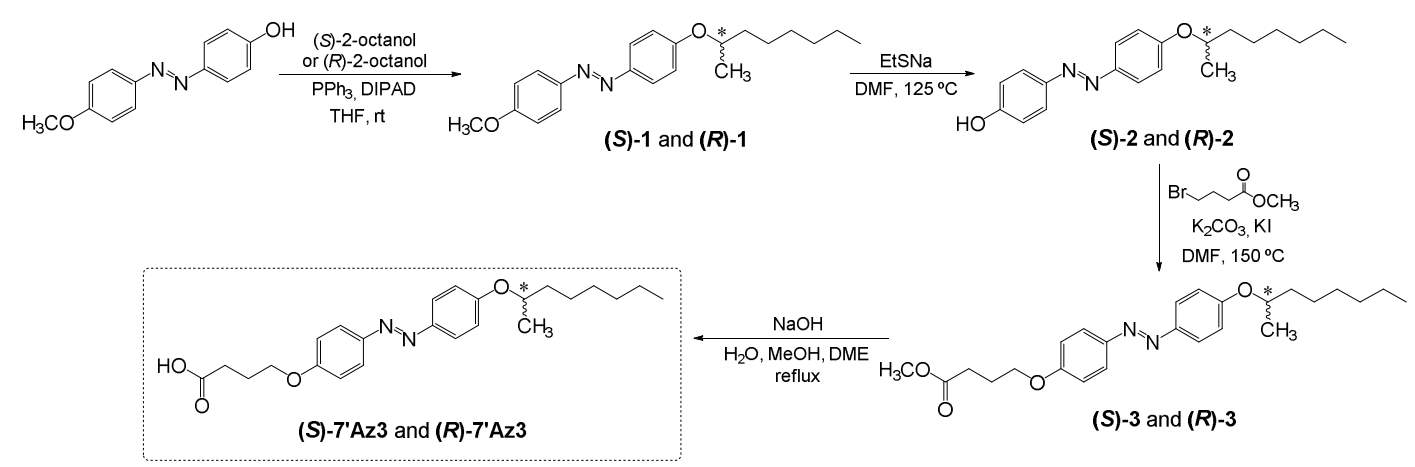

Scheme 1. Synthetic concept for the chiral azo-dyes (R)-7'Az3 and (S)-7'Az3. 
1

2

3

4

5

6

7

8

9

10

11

12

13

14

15

16

17

18

19

20

21

22

23

24

25

26

27

28

29

30

31

32

33

34

35

36

37

38

39

40

41

42

43

44

45

46

47

48

49

50

51

52

53

54

55

56

57

58

59

60 


\section{RESULTS AND DISCUSSION}

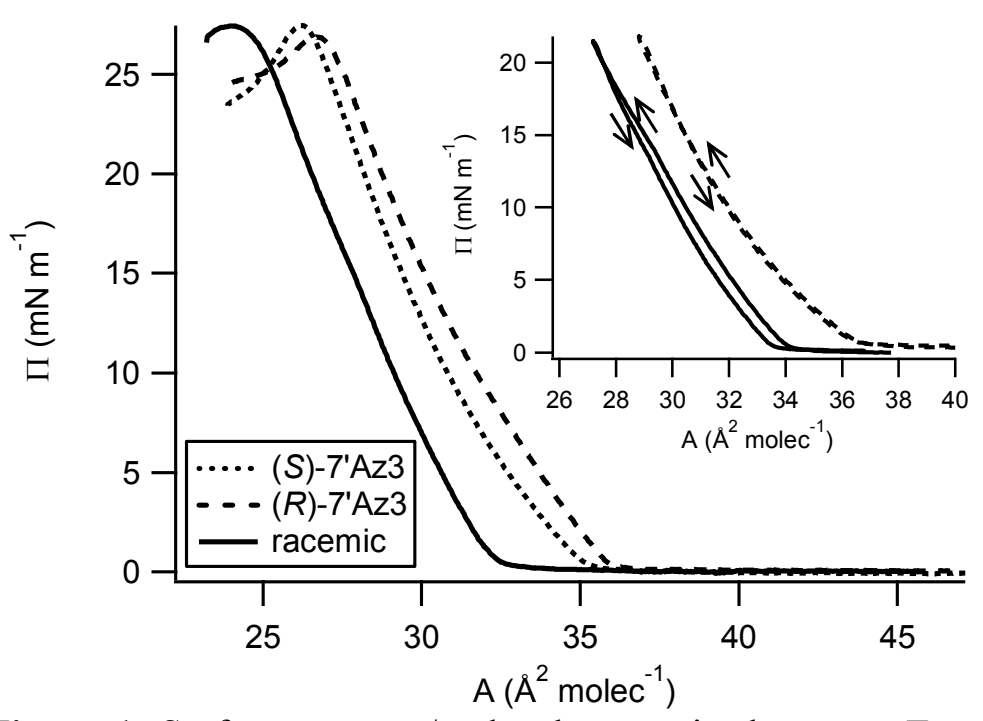

Figure 1. Surface pressure/molecular area isotherms at $\mathrm{T}=25^{\circ} \mathrm{C}$ for monolayers of pure $(S)-7^{\prime} \mathrm{Az3},(R)-7^{\prime} \mathrm{Az} 3$, and for a racemic mixture. All isotherms are the average of five experimental realizations. In the inset, one of the isotherms for the $\mathrm{R}$ enantiomer and one for the racemic mixture are displayed as a compression-expansion cycle.

Langmuir films. We have measured surface pressure/molecular area isotherms for monolayers with the pure enantiomers and for a racemic mixture of the two chiral surfactants (Fig. 1). We have taken great care to ensure that the azobenzene derivatives are in the thermodynamically stable trans form by preparing the spreading solutions under dim red light and by performing the experiments in the dark or under dim red light. We report data for a temperature $\mathrm{T}=25^{\circ} \mathrm{C}$, although no significant differences have been observed for temperatures between $15^{\circ} \mathrm{C}$ and $35^{\circ} \mathrm{C}$. The isotherms of both enantiomers have an observable but small shift in their relative area per molecule values that we attribute to experimental uncertainties. In other words, our observations are compatible with both enantiomers featuring identical thermodynamic behavior, as one 
would expect in the absence of any external chiral influence ${ }^{8}$. Pressure lift-off takes place at around $35 \AA^{2} \mathrm{molec}^{-1}$, comparable to the value obtained for the achiral analogue $8 \mathrm{Az} 3^{19}$, and slightly below the value reported by Haro et al. ${ }^{21}$ for a compound of similar chain length but more distal position of the azobenzene moiety. A compression/expansion cycle shows little hysteresis provided the surface pressure is kept below monolayer collapse, whose signature is the appearance of a local maximum in the monolayer (Fig. 1).

Real time Brewster angle microscopy reveals that the monolayers are isotropic at optical length-scales until collapse takes place below an area per molecule of $27 \AA^{2}$ molec $^{-1}$, approximately. This is consistent with the experiments by Haro et al. where the authors report a featureless monolayer under BAM observations, and is in contrast to the behavior or $8 \mathrm{Az} 3$, which features an ordered expanded phase that was attributed to the tendency of the trans azobenzene hydrophobic tails to form face-to-face H-aggregates on the air/water interface ${ }^{25}$. Nevertheless, we have found no measurable difference in the UV-vis spectrum of enantiomeric and racemic $7^{\prime} \mathrm{Az} 3$ monolayers after LB transfer (Figure S4), observing the signature of H-aggregation (hypsochromic shift in the UVvis reflection spectrum) in both cases. A similar observation has been reported by Haro et al. for monolayers of $(S)-3^{\prime} \mathrm{Az} 7^{21}$, both in-situ and after LB transfer.

In previous experiments with the achiral analogue 8Az3, monolayers of a photostationary mixture of the cis and the trans isomers phase separate at the air/water interface, resulting in the coexistence of optically birefringent sub-millimeter regions rich in the trans isomer surrounded by optically isotropic regions rich in the cis isomer $^{19}$. We have attempted to observe similar phase segregation with $(R / S)-7^{\prime} \mathrm{Az} 3$, both by spreading a room light photostationary cis-trans mixture of either chiral enantiomer or by first spreading a pure trans monolayer followed by irradiation with 
UV light (see Figure S2, supplementary information). Although an expansion following trans to cis isomerization is detected by surface pressure measurements, BAM images reveal a homogeneous monolayer at all times. This might suggest either that cis-trans segregation does not occur for these compounds or that segregation does occur but the arrangement of the two isomers (lateral order and packing density) is too similar to be detected by our imaging equipment. Experimental evidence below with monolayers of a racemic mixture suggests that the latter hypothesis is most likely.

We have also studied monolayers prepared with an equimolar (racemic) mixture of $(R / S)-7^{\prime}$ Az3. Pressure/area isotherm data in Figure 1 reveal that mixed monolayers feature a condensation with respect to an ideal mixture of the pure enantiomers, with a surface excess area of about $2 \AA^{2}$ molec $^{-1}$. Remarkably, BAM images show that this condensation is accompanied by the onset of orientational order in the monolayer, as revealed by the formation of textures with regions of very different reflectivity that changes if the BAM analyzer is rotated. This is a signature of optical birefringence (Figure 2), which is reminiscent of hexatic textures described in long-chain fatty acids ${ }^{26}$ and for the achiral analogue $8 \mathrm{Az} 3$ at high lateral pressures ${ }^{19,}{ }^{27}$.This hypothesis is confirmed below when lateral boundary conditions induce the formation of highly symmetric textures. The average size of the birefringent domains grows significantly with time for the first few minutes and remains unchanged, or changes much more slowly afterwards. Changes in the thermodynamic properties of racemic mixtures of Langmuir monolayers with respect to the pure enantiomers are not unusual ${ }^{4,5,21}$, given the different possible packing arrangements at the air/water interface of pure enantiomers and racemic mixtures ${ }^{7}$. Compression of the racemic monolayer has no significant effect on the observed patterns until a pressure close to $10 \mathrm{mN} \mathrm{m}^{-1}$ is approached. Domains become fragmented and birefringence disappears rather abruptly 
(Fig. 2, B and C). It is puzzling that this loss of birefringence is not accompanied by a detectable feature in the isotherm (Fig. 1), as changes between a disordered and a hexatic phase usually involve a first order phase transition evidenced by a plateau in the isotherm. Moreover, one would expect the surface excess area to decrease when birefringence is lost, as ordering approaches that of the pure enantiomers. In other words, one would expect the isotherm of the racemic mixture to approach that of the pure enantiomers above $10 \mathrm{mN} \mathrm{m}^{-1}$. It remains unclear why this does not happen. A possible explanation would be that the loss of optical contrast is due to a progressive untilting of the hydrophobic chains until they become oriented perpendicularly to the interface. This hypothesis, however, is refuted by experimental evidence below.

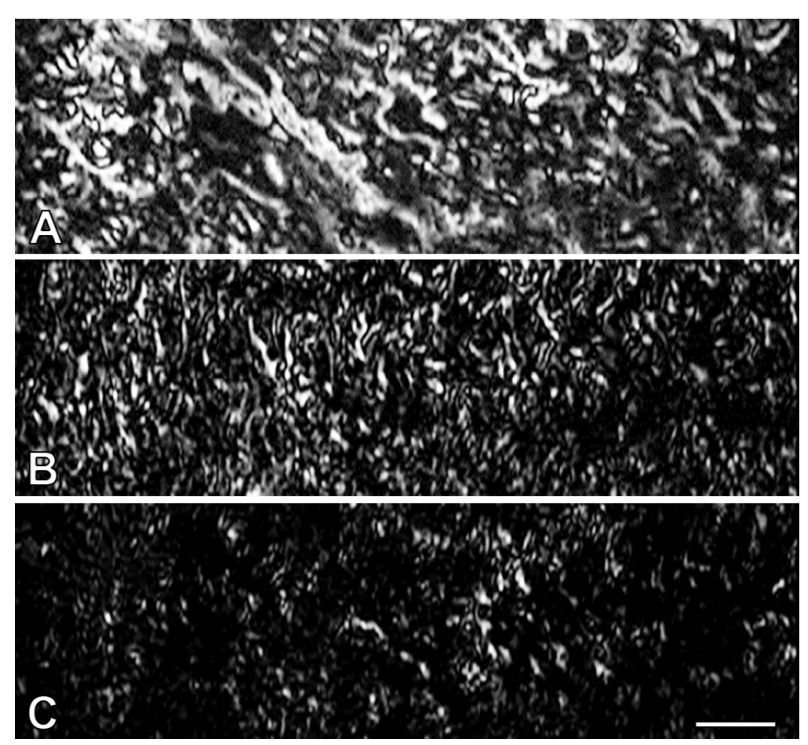

Figure 2. BAM images of a racemic mixture of $(S)-7^{\prime} \mathrm{Az3},(R)-7^{\prime} \mathrm{Az3}$, revealing the onset of orientational order and its degradation upon compression. Lateral pressures are $1.0 \mathrm{mN} \mathrm{m}^{-1}(\mathrm{~A}), 9.7 \mathrm{mN} \mathrm{m}^{-1}(\mathrm{~B})$, and $10.7 \mathrm{mN} \mathrm{m}^{-1}(\mathrm{C})$. The monolayer has relaxed at constant area for 30 minutes in (A). The line segment in (C) is $100 \mu \mathrm{m}$ long.

Similarly to the procedures often employed in the liquid crystal literature, the nanoscale ordering of monolayer mesophases is typically inferred from the microscale 
BAM textures of highly symmetric configurations, which can be obtained in isolated submillimeter domains ${ }^{22}$. Boundaries impose anchoring conditions on the molecular orientation that are topologically incompatible with the nanoscale order, which results in the formation of point defects characteristic of the underlying order. In earlier experiments with the analogue $8 \mathrm{Az} 3^{19}$, we could prepare isolated mesophase domains taking advantage of the spontaneous phase separation of the cis and trans isomeric forms, which results in the formation of pinwheel patterns, characteristic of hexatic phases. Following similar procedures, we generate here mesophase domains by spreading a monolayer with a cis-rich racemic mixture (previous irradiation with UV light of the spreading solution), allowing the slow spontaneous relaxation into the trans isomers and subsequent segregation and self-assembly of the trans-rich mesophase domains. Indeed, BAM images reveal the formation of the pinwheel patterns, organized in sectors of uniform reflectivity (uniform molecular orientation), an unambiguous signature of hexatic order ${ }^{22}$ (Fig. 3). Since the segregation of trans-rich and cis-rich domains is observed for the racemic mixture, the reason why such a segregation was not observed for the pure enantiomers was probably due to a lack of optical reflectivity contrast between the cis-rich and trans-rich domains in the latter case. The analysis of BAM micrographs of the isolated domains can be used to characterize the orientational order of the surfactants. Since the total chain length and the position of the azobenzene moiety in $(R / S) 7^{\prime} \mathrm{Az} 3$ is close to that in $8 \mathrm{Az} 3$, we can use the optical dielectric properties of the latter ${ }^{19}$ to perform calculations in the current system, yielding an estimate for the average tilt angle of about $45^{\circ}$ with respect to the interface normal, and an average tilt oriented along the bisector in each circular sector of an isolated domain (Fig. 3). 

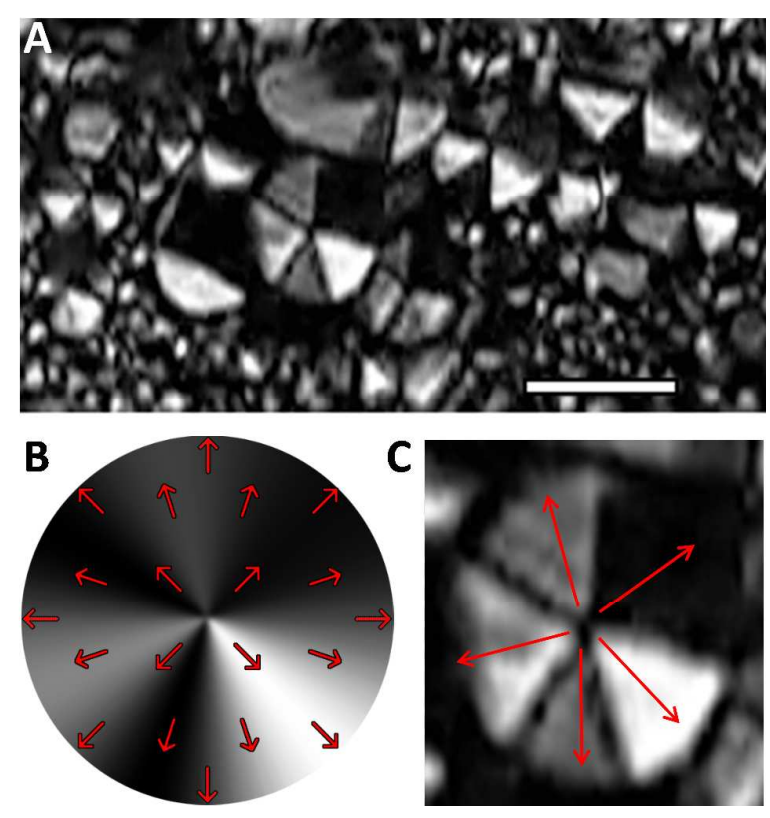

Figure 3. (A) BAM micrograph of trans-rich domains surrounded by a cis-rich matrix in a monolayer containing a racemic mixture of the chiral azobenzene surfactants. An optical analysis enables to relate the digitized reflectivity patterns with the modeled molecular order (B), which enables to obtain the local average in-plane molecular orientation (C). The ruler in (A) is $100 \mu \mathrm{m}$ long.

We can take further advantage of these high symmetry configurations to ascertain whether the loss of contrast upon compression of the hexatic phase in the racemic mixture of $(R / S) 7^{\prime} \mathrm{Az} 3$ is due to transition into a low-birefringence untilted phase (see above). If this were the case, the same structures observed before the loss of contrast at high pressure would be regained once the pressure is lowered before the transition threshold. We proceed by compressing a monolayer with structured domains such as that reported in Fig. 3A until a pressure well above the loss of BAM contrast, and well below the collapse. We subsequently expand the monolayer until BAM contrast is regained. The result is that the recovered birefringent phase is now very fragmented, similar to a freshly spread monolayer, with no sign of the pinwheel patterns observed 
before compression. We thus conclude that the loss of contrast is accompanied with a loss of the hexatic order present at low pressures.

Compression beyond the collapse point results, for all monolayer compositions, in the nucleation and growth of submillimeter domains with multi-layer thickness, as revealed by the large increase in BAM reflectivity. One would expect these films to be tri-layers or, in general, be formed by an odd number of monolayers arranged head-to-head and tail-to-tail in order to always expose the hydrophobic tails to the air. ${ }^{21}$ In fact, UV-vis spectroscopy performed on LB films obtained before and well after the collapse reveal that the reflected light intensity is roughly three times larger for the multilayer film, suggesting that, on average, monolayers collapse into tri-layers. The presence of orientational order inside the collapsed domains is revealed by their optical birefringence, which is made evident by rotating the BAM analyzer (Figures 4 and S3 for observations on LB films). We have qualitatively analyzed a number of collapsed domains obtained for the different system compositions with the purpose of evidencing a signature of heterochiral recognition similar to the one we have reported above for monolayers. Collapsed domains of the two enantiomers have compact shapes, with rough boundaries and an inner texture that often features spiral motifs (see Fig. 4A), although no clear evidence for enantiomorphic configurations is observed when comparing multi-layer domains of the pure enantiomers. For the racemic mixture, collapsed domains are less compact in shape, featuring coexistence between regions with rough edges and inner texture with regions with both smooth edges and inner texture. As we see in the next section, a nanoscale ordering is evidenced in the collapsed domains after their transfer onto solid support. 



Figure 4. BAM micrographs of multi-layer domains prepared with pure $(S)$-3 $^{\prime} \mathrm{Az} 7$ (A), $(R)-3^{\prime} \mathrm{Az} 7(\mathrm{~B})$, and with a racemic mixture of the two (C). Images have been taken at the area per molecule at which lateral pressure exhibits a maximum (onset of collapse). Birefringence in these domains is evident by rotating the BAM analyzer (snapshots of a domain at four different analyzer orientations are shown on the right column next to each image). BAM laser intensity has been attenuated to compensate for the increased reflectivity of these thick films. 
Langmuir-Bodgett films. Further information on the molecular arrangement in the multilayer domains nucleating upon monolayer collapse can be obtained by transferring the films onto solid support and performing atomic force microscopy (AFM) measurements on them. LB transfer took place just after monolayer collapse, maintaining constant the surface pressure during the process. BAM images acquired at the air/water interface prior to LB extraction (Fig. 4) reveal the nucleation and growth of multilayer domains featuring long-range orientational order, which is evidenced by rotating the BAM analyzer. Given that both the typical domain size and their average spacing are larger than the range of our largest AFM scanner $(70 \mu \mathrm{m})$, the ability to properly place the AFM tip on top of a domain is crucial in this case. We have therefore transferred the surfactant films onto engineered silicon substrates (SURF) that allow optical inspection of the thin films and performed AFM scans over regions whose structure is known at optical length scales thanks to the modified AFM header combined with a polarizing reflection microscope (see Experimental Section above). High resolution optical micrographs reveal that the domain morphology is preserved by the LB transfer process, including their birefringent nature, and hint at a nanostructure that could not be resolved by means of BAM imaging (See Figure S3). Since there is no strong bond between the carboxylic group of our surfactant molecules and the $\mathrm{SiO}_{2}$ surface, films tend to desorb and surfactant micro-crystals grow after a few hours. As a result, LB films of the pure enantiomers inspected weeks after their transfer have degraded, and the original domain structure is no longer observable. In the case of films prepared with the racemic mixture, film stability is noticeably enhanced. For instance, in Fig. S3 we report micrographs of a LB film that was eight weeks old. In this case, only modest film degradation is detected. 
Our AFM data clearly reveal that collapsed domains are formed by isolated, micrometer-size elongated multilayer subdomains (Fig. 5) arranged in a nematic-like local orientational order, which is presumably the cause of the detected optical birefringence under BAM observation. In the case of films of the pure enantiomers, the orientation of elongated subdomains changes continuously around the center of the parent domain, while in the case of films of the racemic mixture, the parent domain is organized in regions inside of which elongated subdomains remain roughly parallel. 


\section{A}



B

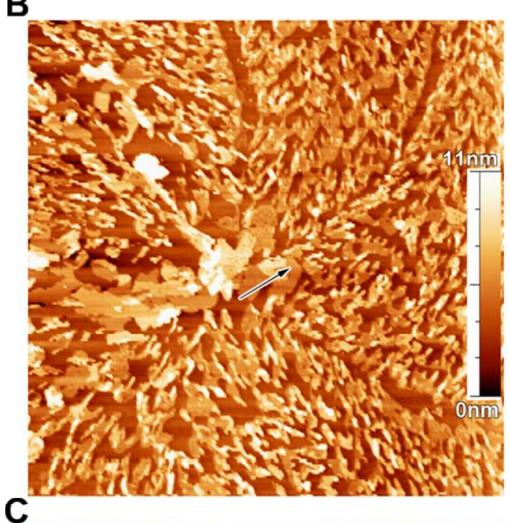

C

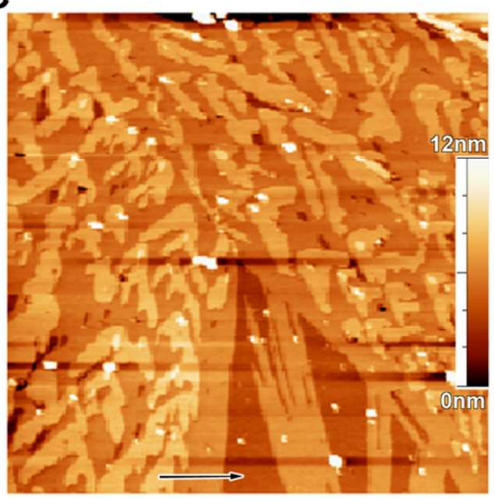



Figure 5. AFM topography scans

of a region near the center of a collapsed domain in LB films on
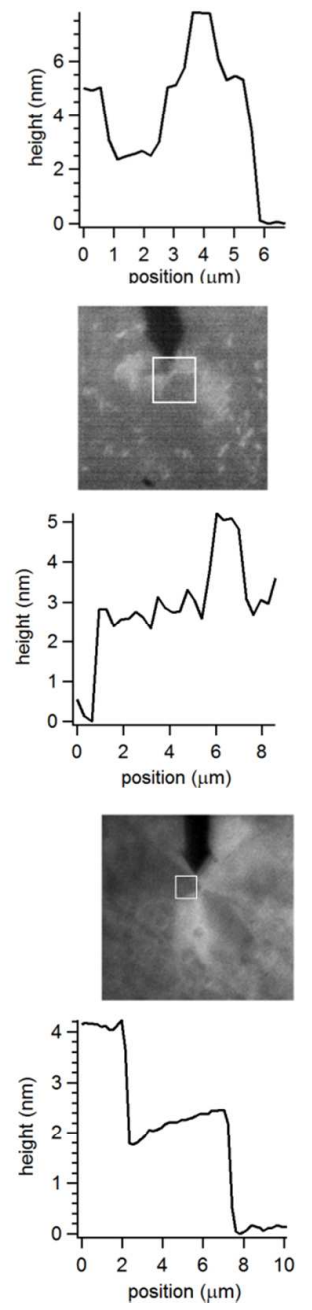
window sizes are (S)-7’Az3, $70 \times 70 \mu \mathrm{m}^{2} \quad(\mathrm{~A}) ; \quad(R)-7^{\prime} \mathrm{Az} 3$, $70 \times 70 \mu^{2}(\mathrm{~B})$; racemic mixture $70 \times 70 \mu^{2}(\mathrm{~B})$; racemic mixture $\mu \mathrm{m}^{2}(\mathrm{C})$.

SURF substrates of chiral azobenzene surfactants at different compositions. To the right of each AFM image an optical micrograph shows the location of the scanned region, marked with a white square, as observed by in-situ polarizing reflection microscopy. The height profile along a linear path, marked by an arrow on the AFM image, is also included to the right of the image. Film composition and scanned $\mu \mathrm{m}(\mathrm{C})$ 


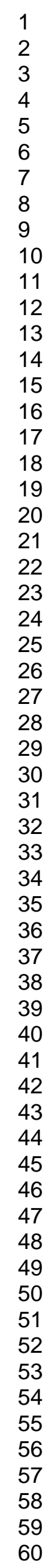


We have measured the thickness profile across the main features observed on AFM scans of the LB films (Fig. 5). For the films of the pure enantiomers, most elongated domains have an average thickness of $5.0 \mathrm{~nm}$ above the lowest level. There are a few scattered features whose thickness is either $2.5 \mathrm{~nm}$ or $7.5 \mathrm{~nm}$ above the baseline level, indicating a characteristic step size of $2.5 \mathrm{~nm}$. For the films prepared with the racemic mixure, scattered elongated domains are $4.0 \mathrm{~nm}$ thicker than the baseline level, and they stand $2.0 \mathrm{~nm}$ above a uniform continuous region that stands $2.0 \mathrm{~nm}$ above the baseline level (Fig. 5). In order to interpret these measurement, we have estimated the length of the chiral azobenzene surfactant molecules, which is approximately $2.5 \mathrm{~nm}^{28}$. The most likely arrangement for the collapsing monolayer is to spontaneously organize in the socalled Y type film ${ }^{28}$, in which stacked monolayers arrange in a head-to-head or tail-totail fashion, always exposing hydrophobic tails to the air. Consequently, one expects that the baseline region on the LB films, which have been transferred by a single upstroke, corresponds to the first monolayer, and that subsequent steps correspond to bilayers. This is consistent with UV-vis measurements performed on LB films obtained for fully collapsed monolayers (Fig. S4). Data indicate an absorbance roughly three times large for the collapsed monolayers than for the single monolayer LB films, indeed suggesting that the former are trilayers on average. In order to understand the measured bilayer thickness when compared to the estimated molecular length we need to take into account that molecules are most likely tilted with respect to the interface normal, as suggested by the optical birefringence of collapsed domains (birefringence is maximal when the optical axis of the material is perpendicular to the light path). A final aspect that must be taken into account to interpret molecular arrangement inside collapsed domains is the insertion of hydrophobic chains in adjacent monolayers, which has been suggested to be significant in similar systems ${ }^{21}$. In the case of LB films of the racemic 
mixture, one can take as a reference value the tilt angle that we have estimated from the analysis of BAM micrographs of hexatic domains (see above), namely $\theta=45^{\circ}$. With this, the thickness of a bilayer, ignoring chain insertion, would be $3.6 \mathrm{~nm}$. In consequence, the measured bilayer thickness (step height reported above) would imply a chain insertion of about $1.6 \mathrm{~nm}$ for films of the racemic mixture, and $1.1 \mathrm{~nm}$ in the case of films of the pure enantiomers. The increased chain insertion in racemic films is consistent with the enhancement in the film stability reported above. Chain insertion also favors interaction between molecules in adjacent monolayers, as is evidenced by the bathochromic shift observed in UV-vis spectra (Fig. S4). 


\section{CONCLUSIONS}

We have reported studies of the assembly behavior of chiral azobenzene surfactants at the air/water interface. While the presence of the chiral center in the hydrophobic chain hampers the ability of trans isomers of these compounds to form mesophases (compared to achiral analogues), a significant heterochiral recognition is observed in monolayers prepared with the racemic mixture. This is evidenced during Brewster angle microscopy observations, which reveal the formation of a mesophase. By preparing monolayers in the form of isolated trans-azobenzene rich domains, the nanoscale ordering couples with the anisotropic boundary conditions at the domain boundary, resulting in textures that unambiguously indicated the formation of a hexatic phase. Following previously described methods we have been able to characterize the orientational order at the mesoscale, in particular the average orientation of tilted hydrophobic chains. Compression of the hexatic racemic monolayer leads to a transition into a non-birefringent phase. Although hexatic order is regained upon expansion, the range of the tilt-orientational order is severely diminished. Further studies involving grazing-incidence $\mathrm{x}$-ray diffraction and spectroscopic techniques would be needed to propose a model that fully characterizes the arrangement of the two enantiomers in the hexatic lattice of the mesophase, to understand the changes that take place upon compression from an ordered into a disordered phase, and to compare the molecular arrangement of the disordered racemic phase with that in the disordered phases observed for pure enantiomers. For all compositions, high compression leads to film collapse into ordered stacks of odd-number monolayers, with the formation of submillimeter birefringent crystallites featuring an inner nanostructure, as revealed by 
AFM studies of LB-transferred films. Under these conditions, interlayer penetration is enhanced for the racemic mixture.

The observed heterochiral recognition effects could be explored under adsorption at different interfaces. Recently, Iavicoli et $a .^{29}$ reported that the presence or absence of spontaneous resolution of a racemate of self-assembling surfactants is dramatically dependent on the nature of the support surface. In our case, the adsorption of the chiral azobenzene surfactants could be studied at the solution/solid interface during the formation of self-assembled monolayers, or at the liquid crystal/water interface, which is known to amplify changes in the mesoscale ordering of adsorbed species ${ }^{30}$. In the latter context, structured monolayers of achiral analogues of these compounds have also been employed successfully to pattern substrates via the LB technique showing that this can be a new strategy to structure liquid crystal devices at the microscale ${ }^{31}$. In fact, the Langmuir-Blodgett technique has recently been acknowledged to offer a new bottom-up approach to pattern surfaces with large areas with well-defined microstructures, which may be employed as an interface between biological systems and material science ${ }^{32}$. 


\section{ASSOCIATED CONTENT}

Supporting Information. Diagram with modified AFM header to enable optical resolution of heterogeneous LB film. Monolayer compression isotherm due to trans-tocis isomerization. Polarizing optical micrographs of LB films on SURF substrates. This material is available free of charge via the Internet at http://pubs.acs.org.

\section{AUTHOR INFORMATION}

Corresponding Author

*E-mail: jignes@ub.edu

\section{Funding Sources}

A. P.-C., R. A., and J. I.-M. acknowledge support from MICINN (Project FIS201021924-C02-01) and by DURSI (Project 2009 SGR 1055). A. P.-C. acknowledges support from Generalitat de Catalunya through grant 2011-FI-B00131. D. V. and J. G.A. acknowledge support from MICINN (Project CTQ2012-36074).

\section{Notes}

The authors declare no competing financial interest.

\section{ACKNOWLEDGMENT}

We thank J. Legurburu for assistance during the experiment, and F. Sagués and Josep Claret for helpful discussions.

\section{Supporting Information Available}

Detailed synthesis and characterization of the organic compounds, schematics of the modified AFM setup, photoresponse of the monolayers, optical micrographs evidencing the birefringence of the collapsed monolayer domains. This information is available free of charge via the Internet at http://pubs.acs.org/. 





1

2

3

4

5

6

7

8

9

10

11

12

13

14

15

16

17

18

19

20

21

22

23

24

25

26

27

28

29

30

31

32

33

34

35

36

37

38

39

40

41

42

43

44

45

46

47

48

49

50

51

52

53

54

55

56

57

58

59

60
Table of contents only

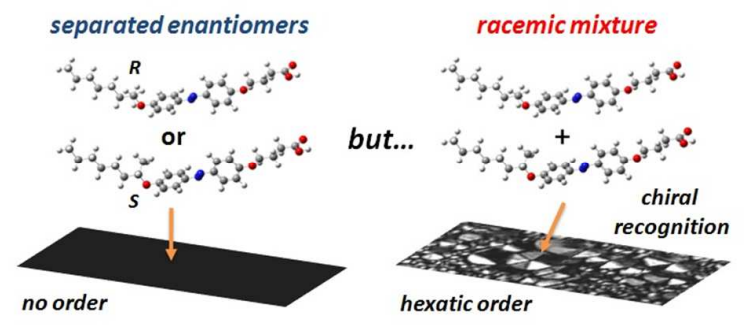




\section{REFERENCES}

1. Vollhardt, D.; Nandi, N.; Banik, S. D., Nanoaggregate shapes at the air/water interface. Phys Chem Chem Phys 2011, 13, (11), 4812-29.

2. Petty, M. C., Langmuir-Blodgett films : an introduction. Cambridge University Press: Cambridge ; New York, 1996; p xviii, 234 p.

3. Ariga, K.; Michinobu, T.; Nakanishi, T.; Hill, J., Chiral recognition at the airwater interface. Current Opinion in Colloid \& Interface Science 2008, 13, (1-2), 23-30.

4. Arnett, E. M.; Harvey, N. G.; Rose, P. L., Stereochemistry and molecular recognition in "two dimensions". Acc Chem Res 1989, 22, (4), 131-138.

5. Kuzmenko, I.; Kjaer, K.; Als-Nielsen, J.; Lahav, M.; Leiserowitz, L., Detection of Chiral Disorder in Langmuir Monolayers Undergoing Spontaneous Chiral Segregation. J Am Chem Soc 1999, 121, (12), 2657-2661.

6. Mamdouh, W.; Uji, I. H.; Dulcey, A. E.; Percec, V.; De Feyter, S.; De Schryver, F. C., Expression of molecular chirality and two-dimensional supramolecular selfassembly of chiral, racemic, and achiral monodendrons at the liquid-solid interface. Langmuir 2004, 20, (18), 7678-85.

7. Weissbuch, I.; Leiserowitz, L.; Lahav, M., Molecular recognition in chiral and non-chiral amphiphilic self-assemblies at interfaces. Current Opinion in Colloid \& Interface Science 2008, 13, (1-2), 12-22.

8. Nandi, N.; Vollhardt, D., Effect of molecular chirality on the morphology of biomimetic langmuir monolayers. Chem Rev 2003, 103, (10), 4033-76.

9. Jimenez-Millan, E.; Giner-Casares, J. J.; Martin-Romero, M. T.; Brezesinski, G.; Camacho, L., Chiral Textures inside 2D Achiral Domains. J Am Chem Soc 2011.

10. Ignes-Mullol, J.; Claret, J.; Sagues, F., Star defects, boojums, and cardioid droplet shapes in condensed dimyristoylphosphatidylethanolamine monolayers. Journal of Physical Chemistry B 2004, 108, (2), 612-619.

11. Nandi, N.; Vollhardt, D., Chiral discrimination and recognition in Langmuir monolayers. Current Opinion in Colloid \& Interface Science 2008, 13, (1-2), 40-46.

12. Percec, V.; Asandei, A. D.; Zheng, Q., Chiral recognition in molecular and macromolecular pairs of (S)- and (R)-1-cyano-2-methylpropyl-4'- $\{[4-(8-$ vinyloxyoctyloxy)benzoyl]oxy\} biphenyl-4- carboxylate enantiomers. Journal of Polymer Science Part A: Polymer Chemistry 2000, 38, (19), 3631-3655.

13. Percec, V.; Oda, H., Heterochiral Interactions in Molecular and Macromolecular Pairs of Liquid Crystals of (R)- and (S)-2-Fluoro-4-methylpentyl 4'-((8(Vinyloxy)octyl)oxy)biphenyl-4-carboxylate Enantiomers. Macromolecules 1994, 27, (20), 5821-5832.

14. Zhu, X.; Li, Y.; Duan, P.; Liu, M., Self-assembled ultralong chiral nanotubes and tuning of their chirality through the mixing of enantiomeric components. Chemistry 2010, 16, (27), 8034-40.

15. Cao, H.; Zhu, X.; Liu, M., Self-Assembly of Racemic Alanine Derivatives: Unexpected Chiral Twist and Enhanced Capacity for the Discrimination of Chiral Species. Angew Chem Int Ed Engl 2013.

16. Seki, T.; Sakuragi, M.; Kawanishi, Y.; Tamaki, T.; Fukuda, R.; Ichimura, K.; Suzuki, Y., "Command surfaces" of Langmuir-Blodgett films. Photoregulations of liquid crystal alignment by molecularly tailored surface azobenzene layers. Langmuir 1993, 9, (1), 211-218. 
17. Ignés-Mullol, J.; Claret, J.; Reigada, R.; Sagués, F., Spread monolayers: Structure, flows and dynamic self-organization phenomena. Physics Reports 2007, 448, (5-6), 163-179.

18. Tabe, Y.; Yamamoto, T.; Yokoyama, H., Photo-induced travelling waves in condensed Langmuir monolayers. New Journal of Physics 2003, 5, 65-65.

19. Crusats, J.; Albalat, R.; Claret, J.; Ignes-Mullol, J.; Sagues, F., Influence of temperature and composition on the mesoscopic textures of azobenzene Langmuir monolayers. Langmuir 2004, 20, (20), 8668-8674.

20. Petit-Garrido, N.; Claret, J.; Ignes-Mullol, J.; Sagues, F., Stirring competes with chemical induction in chiral selection of soft matter aggregates. Nat Commun 2012, 3, 1001.

21. Haro, M.; del Barrio, J.; Villares, A.; Oriol, L.; Cea, P.; Lopez, M. C., Supramolecular architecture in Langmuir and Langmuir-Blodgett films incorporating a chiral azobenzene. Langmuir 2008, 24, (18), 10196-203.

22. Fischer, T. M.; Bruinsma, R. F.; Knobler, C. M., Textures of surfactant monolayers. Physical Review E 1994, 50, (1), 413-428.

23. Horcas, I.; Fernandez, R.; Gomez-Rodriguez, J. M.; Colchero, J.; GomezHerrero, J.; Baro, A. M., WSXM: a software for scanning probe microscopy and a tool for nanotechnology. Rev Sci Instrum 2007, 78, (1), 013705.

24. Amaike, M.; Kobayashi, H.; Sakurai, K.; Shinkai, S., Novel Attempts to Change the Colour of Dye Molecules Utilizing the Aggregation Mode of Saccharides. Supramolecular Chemistry 2002, 14, (2-3), 245-253.

25. Pedrosa, J.-M.; Romero, M. T. M.; Camacho, L.; Möbius, D., Organization of an Amphiphilic Azobenzene Derivative in Monolayers at the Air-Water Interface. The Journal of Physical Chemistry B 2002, 106, (10), 2583-2591.

26. Ignes-Mullol, J.; Schwartz, D. K., Molecular orientation in Langmuir monolayers under shear. Langmuir 2001, 17, (10), 3017-3029.

27. Durbin, M. K.; Malik, A.; Richter, A. G.; Yu, C. J.; Eisenhower, R.; Dutta, P., Ordered Phases in Langmuir Monolayers of an Azobenzene Derivative. Langmuir 1998, 14, (4), 899-903.

28. Hanwell, M. D.; Curtis, D. E.; Lonie, D. C.; Vandermeersch, T.; Zurek, E.; Hutchison, G. R., Avogadro: An advanced semantic chemical editor, visualization, and analysis platform. $J$ Cheminform 2012, 4, (1), 17.

29. Iavicoli, P.; Xu, H.; Keszthelyi, T.; Telegdi, J.; Wurst, K.; Van Averbeke, B.; Saletra, W. J.; Minoia, A.; Beljonne, D.; Lazzaroni, R.; De Feyter, S.; Amabilino, D. B., Organization of the enantiomeric and racemic forms of an amphiphilic resorcinol derivative at the air-water and graphite-1-phenyloctane interfaces. Chirality 2012, 24, (2), 155-66.

30. Bai, Y.; Abbott, N. L., Recent advances in colloidal and interfacial phenomena involving liquid crystals. Langmuir 2011, 27, (10), 5719-38.

31. Petit-Garrido, N.; Trivedi, R.; Ignés-Mullol, J.; Claret, J.; Lapointe, C.; Sagués, F.; Smalyukh, I., Healing of Defects at the Interface of Nematic Liquid Crystals and Structured Langmuir-Blodgett Monolayers. Physical Review Letters 2011, 107, (17), 177801 .

32. Chen, X.; Lenhert, S.; Hirtz, M.; Lu, N.; Fuchs, H.; Chi, L., Langmuir-Blodgett patterning: a bottom-up way to build mesostructures over large areas. Acc Chem Res 2007, 40, (6), 393-401. 




